\title{
Interview With Lars Peter Hansen
}

1. Eric Ghysels and Alastair Hall (Editors): How did you come to be interested in estimation based on moment conditions?

Lars Peter Hansen (L.P.H.): As a graduate student at the University of Minnesota, I had the opportunity to take classes from Chris Sims and Tom Sargent. Both emphasized the idea that dynamic econometric models should be viewed as restrictions on stochastic processes. Sims's classroom development of largesample econometrics broke with the more conventional view of the time of focusing on models with exogenous regressors or instrumental variables (IVs). His work on causality had emphasized the testable restrictions on stochastic processes of the notion of strict exogeneity. The idea of embedding estimation and testing problems with a stochastic process framework proved to be a very useful starting point. Although this is common in econometrics today, it was not at the time I was a graduate student.

I was exposed to nonlinear estimation and testing problems in Sims's graduate course. Sims loved to experiment with new material and proofs in class, and I learned much from filling in details and some holes in lecture notes. His nonconventional teaching style was valuable for me.

While I was working on my dissertation on exhaustible resources, I became interested in central limit theory for temporally dependent processes. This interest predated the excellent book by Hall and Heyde (1980). Sims had made reference to work by Scott (1973), but the central paper for me was that of Gordin (1969). (Scott indeed cites Gordin, which is how I became familiar with that work.) Gordin showed how to construct a martingale approximation to a wide class of stationary ergodic processes. It thus extended the applicability of earlier martingale central limit theory by Billingsley (1961) and others to processes with a rich temporal dependence structure.

2. Editors: With hindsight, it can be seen that your paper (Hansen 1982) has had considerable influence on the practice of econometrics. What was your perspective on the work at the time? Can you also tell us about the initial reaction of others (including referees!) to your paper?

L.P.H.: I originally wrote a paper on least squares estimation with temporally dependent disturbances. The motivation for this paper was simple. As an editor of Econometrica, Sims was handling a paper by Brown and Maital (1981) on assessing the efficiency of multiperiod forecasts. The multiperiod nature induced temporal dependence in the disturbance term. Sims urged me to proceed quickly to get something written up as a paper that could be cited and used. Hodrick (my colleague at Carnegie Mellon at the time) made me aware of similar problems in the literature on the study for forward exchange rates as predictors of future spot rates. When the forward contract interval exceeds the sampling interval, temporal dependence is induced. Given the serial correlation in the disturbance term, many applied researchers at the time thought the right thing to do was to use a standard generalized least squares (GLS)-type correction. In fact, while least squares remains consistent, the lack of strict exogeneity of the regressors prevents GLS from being consistent. This tripped up several researchers and was the impetus for my original least squares paper. In addition to the Brown and Maital paper, which with Sims's influence proceeded correctly, Hodrick quickly informed authors of the papers submitted to the Journal of Political Economy that the GLS style correction that they had used was invalid.

My least squares paper was rejected at Econometrica because it failed to be ambitious enough. This irritated me and made me restructure the arguments in much greater generality. I had seen recent lecture notes of Sims that described a family of generalized method of moments (GMM) estimators, indexed by a matrix that selected which moment conditions to use in estimation. Sims treated time series applications, but with a focus on martingale difference disturbances. I adopted this formulation to present a more general central limit approximation for estimators with Sims's encouragement. I learned subsequently from a referee that the selection matrix idea had been used by Sargan $(1958,1959)$ in studies of linear and nonlinear instrumental variables estimators. I was a bit embarrassed that I had not cited the very nice Sargan (1958) paper in my original submission. This paper provided the impetus for my analysis of the limiting distribution of sample moment conditions evaluated at parameter estimators.

Since previous referees complained about my casual treatment of consistency, I added some specificity by using a quadratic form construction as in the statistics literature on minimum chi-squared estimation and in Amemiya's $(1974,1977)$ treatments of nonlinear two- and three-stage least squares. While developing consistency results, I became interested in the role of compactness in consistency arguments and explored alternative ways of justifying consistency based on tail characterizations of the objective functions.

3. Editors: Your papers with Ken Singleton in Econometrica (Hansen and Singleton 1982) and Bob Hodrick in the Journal of Political Economy (Hansen and Hodrick 1980) were very influential in demonstrating the potential power of GMM in applications. Can you tell us how these collaborations came about? 
L.P.H.: Bob Hodrick and I began our discussion of econometric issues when I described to him the pitfalls in applying GLS in econometric equations that come from multiperiod forecasting problems. He immediately showed me working papers in the literature on forward exchange rates in which GLS was applied as a remedy for serial correlation. At the same time he was educating people in international finance, he and I began working on our own analysis of forward exchange rates by applying least squares and adjusting the standard errors.

Bob Hodrick was at Carnegie Mellon when I arrived there. Ken Singleton came to Carnegie Mellon after me, and after I had written my GMM paper. Both of us knew Hall's work on consumption (Hall 1978), and Ken came back from a conference after hearing an underappreciated paper by Grossman and Shiller. A stripped-down version of this paper was subsequently published in the American Economic Review (Grossman and Shiller 1981). Singleton suggested that the consumption Euler equation could be fruitfully posed as a conditional moment restriction and our collaboration began. After I arrived at Chicago, Jim Heckman showed me MaCurdy's microeconomic counterpart to Hall's paper (MaCurdy 1978). Ken's and my second (and empirically more interesting) paper published in the Journal of Political Economy (Hansen and Singleton 1983) came about by our construction of a maximum likelihood counterpart to the GMM estimation in our Econometrica paper. Given the more transparent nature of how predictability had implications measuring and assessing risk aversion, this project became more ambitious and took on a life of its own.

4. Editors: What is your perspective on how GMM fits into the wider literature on statistical estimation?

L.P.H.: I spent some time on this question in my recent contribution to the Encyclopedia of the Social and Behavioral Sciences (Hansen 2002). The quadratic-form criterion that I used to formulate consistency certainly has its origins in the minimum chisquared estimators used to produce estimators that are statistically efficient and computationally tractable. An interesting difference is that GMM estimators are often used to study models that are only partially specified, whereas the earlier statistics literature provided computationally attractive estimators for fully specified models. Implicit in many GMM applications is a semiparametric notion of estimation and efficiency, in contrast to minimum chi-squared estimation. There is a more recent related statistics literature on estimating equations, with time series contributions by Godambe and Heyde (1987) and others. This literature was developed largely independently of the corresponding literature in econometrics, but its focus has been primarily on martingale estimating equations. My aim was in part to use Gordin (1969)'s martingale approximation device to adopt a more general starting point.
5. Editors: In the mid-1980s, you worked on the problem of characterizing the optimal instrument in generalized IV estimation. What led you to work on this problem and what is your perspective on this literature today?

L.P.H.: I worked on this problem because many applications of GMM estimation were motivated by conditional moment restrictions or had the following time series structure. Any time an IV existed, lags of the IVs were also valid instruments. As in my other work, I was particularly interested in time series problems. Strict exogeneity of IVs also allows for leading values of IVs to be valid instruments, but this orthogonality was not in my econometric formulation. Prior to my GMM paper, Robert Shiller (1972) had emphasized that in rational expectations models, omission of conditioning information gave rise to orthogonal disturbance. In a time series problem, this Shiller notion of an error term often had the feature that this error term was orthogonal to arbitrary past values of variables in a conditioning information set used by an econometrician. This same argument will not work for arbitrary leading values of the conditioning information, because economic agents will not have seen these values.

My initial work in this area (Hansen 1985) used martingale approximations to give lower bounds on the efficiency of a class of feasible GMM problems. In formulating this problem, I had to extend the selection approach of Sargan, Sims, and others to cases in which the number of underlying moment conditions is infinite. This is a natural framework within which to think about estimation problems in which nonlinear functions of IVs are valid IVs and particularly for time series problems in which lagged values of IVs remain valid instruments. This gave rise to an explicitly infinite-dimensional family of GMM estimators.

Attaining this efficiency, especially in a time series problem, is more problematic. There is a need asymptotically to use information arbitrarily far into the past. While I was working in this area, I became aware of closely related work in engineering aiming to construct online estimators that are easy to compute (see, e.g., Soderstrum and Stoica 1983). Some of their calculations were similar to mine. Also, Gary Chamberlain wrote his very nice paper (Chamberlain 1987) on semiparametric efficiency bounds based on conditional moment restrictions parameterized in terms of a finitedimensional vector and posed in an i.i.d. context.

After my initial characterizations, Singleton and I took a stab at implementation in linear models using Kalman filtering (see Hansen and Singleton 1991; 1996). In many of our examples, we often found only very modest gains in even asymptotic efficiency to using the more fancy procedure over ad hoc choices of variables. While a co-editor at Econometrica, I handled one of Whitney Newey's papers (Newey 1990) that, in an i.i.d. context, attained the efficiency bound. In the sampling experiments that he studied, the efficiency gains were often small, and sometimes the 
actual construction of a nonparametric estimator of an efficient IV undermined the efficiency gains in finite samples. Of course, characterization of the efficiency bound was needed to reach this conclusion.

It is interesting that subsequently, West and Wilcox (1996) reached a rather different conclusion and produced interesting examples in the study of economic time series where the use of the efficiency bounds were of practical value, and they devised some estimation methods that appear to work quite well.

There has been a variety of other interesting and related research on choice of moment conditions to use in actual estimation. Complementary work of Andrews (1999) and Hall and Inoue (2001) considered the selection of which moment conditions to use in parameter estimation. These papers addressed the important practical problem of how to limit the number of moment conditions used in estimation, by excluding irrelevant and invalid ones using adaptive methods.

6. Editors: Method of moments is commonly used now in the estimation of diffusions. Do you expect that this line of research will continue, or do you think that the various simulation-based maximum likelihood estimators will become more widely used?

L.P.H.: My interest in GMM estimation has been primarily to achieve partial identification. That is, suppose the aim of the econometric exercise is to extract a piece of say a fully specified dynamic general equilibrium model. Thus the semiparametric language that Chamberlain emphasized in his work is appropriate. My work with Scheinkman on estimation of nonlinear continuous-time models (Hansen and Scheinkman 1995) and the subsequent work with Conley and Luttmer (Conley, Hansen, Luttmer, and Scheinkman 1997) is best viewed in this light. The idea is that the model is not designed to fit everything, but that there are certain data features such as the long-run stationary distribution that are the appropriate targets. Both of these papers also explore misspecification that takes the form of an exogenous subordination process in the spirit of Clark's fundamental work (Clark 1973).

Of course, partial identification while it may be more realistic, is not a fully ambitious research goal. For many purposes, more is needed. But once a dynamic model is fully specified in a parametric way, maximum likelihood methods and their Bayesian counterpart become attractive. It may be that for numerical reasons, as in Pearson's original work and the subsequent extensive literature on minimum chisquared estimation, method of moments methods are attractive alternatives to likelihood-type estimators. It has been interesting, however, to watch the development of numerical Bayesian methods, methods that make prior sensitivity analyses feasible. The Bayesian problem based on integrating or averaging is numerically simpler than hill-climbing to find a maximum in many circumstances.
7. Editors: In your paper written with Heaton and Yaron in Journal of Business \& Economic Statistics (Hansen, Heaton, and Yaron 1996), you proposed the continuousupdating GMM estimator. Can you tell us something about the background of this paper?

L.P.H.: Initial implementation of GMM estimators in terms of quadratic form minimization involved two-step approaches or iterated approaches. An initial consistent estimator was produced and used to estimate an efficient weighting matrix designed so that the objective has the minimum chi-squared property. In the first-order asymptotic theory, weighting matrix estimation is placed in the background, because all that is needed is a consistent estimator. Stopping after one iteration or continuing are both options.

It was interesting, though, that when Sargan sought to compare IVs estimators to limited information maximum likelihood (LIML) estimators, he produced a depiction of the LIML estimator as an IV-type estimator in which the variance for the structural disturbance term is estimated at the same time the underlying parameters are estimated (Sargan 1958). He essentially concentrated out this variance in terms of the underlying parameters, and produced what can be thought of as a continuous-updated weighting matrix estimator. Under conditional homoscedasticity and a martingale difference structure for the disturbance term, the optimal weighting matrix is the product of the disturbance term variance and the second moment of the IVs. By using the sample secondmoment matrix of the IV and the sample variance of the disturbance term expressed as a function of the unknown parameter vector, in a effect a continuousupdating weighting matrix is produced.

A very similar approach is one of the ways that the minimum chi-squared estimator was implemented for multinomial models. The counterpart to the weighting matrix could be fully parameterized in terms of the multinomial probabilities. In this case the weighting matrix can be produced without reference to the data, but only a function of the unknown parameters. This is because the multinomial model is a full model of the data, while Sargan considered only a partially specified model.

In the more general GMM context with a quadratic form-type objective, it turned out to be quite easy to implement Sargan' approach, except that the simple separation between the disturbance term and the vector of IVs can no longer be exploited. Instead, one constructs the long-run covariance matrices of the function of the data and parameter vector used to produce the parameterized moment condition.

I became interested in this estimator in part to sidestep the issue of whether to iterate on the weighting matrix or not and in part because the sensitivity of both the two-step and iterated estimator to what seemed like arbitrary normalizations. For instance, if one takes the original function of the data $x_{t}$ and a 
hypothetical parameter vector $\beta$ and scales it by some arbitrary function of $\beta$, the moment conditions are preserved. The two-step estimator will be sensitive to this transformation, but the continuous-updating estimator typically will not be. Moreover, in GMM estimation problems that Marty Eichenbaum and I encountered (Eichenbaum and Hansen 1990), we found that in one depiction of the moment conditions, there might be degenerate solutions at the boundary of the parameter space. The continuous-updating estimator would not reward these parameter values, because the degeneracy would cause the weighting matrix to diverge. In contrast, a two-step estimator could easily end up at these often-uninteresting parameter configurations.

In our original paper on continuous-updated GMM estimation, Heaton, Yaron and I found in our Monte Carlo experiments that we tended to replicate what was known in the simultaneous equations literature. The continuous-updating estimator was close to being median unbiased, but it had fat tails. The weighting matrix adjustment often leads to problems in which the objective function itself can be flat in the tails. We found that inference methods based on studying the shape and degradation of the objective function were more reliable than computing quadratic approximations. One formal reason for why this can be true is provided in the paper by Stock and Wright (2000), which studies moment conditions with weak information.

I have found the recent work by Newey and Smith (2000) and Bonnal and Renault (2000) to be very interesting. These papers show how to nest the continuous-updated estimator into a class of estimators that includes empirical likelihood. Newey and Smith (2000), in particular, use second-order asymptotic theory to study this class of estimators. They characterize the advantages in using empirical likelihood to produce parameter estimates when the data are i.i.d. My own interest in the continuous-updating GMM estimator is not so much as a method for producing point estimates, but more as a method of making approximate inference.

8. Editors: A lot of work has focused on estimation of the optimal weighting matrix, which lead to the socalled HAC (heteroskedastic and autocorrelation consistent) estimators. In joint work with Ravi Jagannathan, you also advocate the use of weighting matrices that are suboptimal from a statistical point of view, but have desirable properties in financial applications. What are your thoughts on the issue of weighting matrices?

L.P.H.: The optimal weighting matrix that you refer to is obtained by asking a statistical question. Given that a finite set of moment conditions are satisfied, what is the most efficient linear combination to use in estimating a parameter vector of interest. Ravi and I (Hansen and Jagannathan 1997) and also John Heaton, Erzo Luttmer, and I (Hansen, Heaton, and Luttmer 1995) were interested in a different question.
Suppose that, strictly speaking, the model is misspecified. How might you pick a good model among the parameterized family of models? How does the performance of a parameterized family compare to other models? This leads to a rather different view. Under misspecification, statistical efficiency is put to the side. It becomes a "higher-order" issue. We explore the question of weighting matrix selection in an assetpricing environment and pose the problem as one in which the aim is to keep pricing errors small. The target of the estimation problems of necessity comes to the forefront in this exercise.

Consider an idealized Bayesian decision problem, but suppose that the likelihood function is misspecified. One cannot separate this problem into the following two problems. Find the correct posterior distribution for the parameter vector and then solve the decision problem by minimizing the loss function averaging over the posterior. In the presence of misspecification, we cannot separate the statistician's problem of finding the posterior from that of the decision maker who wishes to use the parameter vector in practice.

Consider now the fiction of an infinite sample. In a GMM context, the weighting matrix that you refer to dictates how important each of the pricing error equations is in pinning down the parameter estimate. However, under correct specification, this choice does not alter the parameter vector that, say, minimizes the population quadratic criterion. Under misspecification, the choice of weighting matrix changes parameter values as you change the weighting matrix, but this also changes the performance criterion. In an asset-pricing context, different weighting matrices alter the importance of pricing errors coming from alternative securities. Parameter choices that lead to moment conditions because of a large covariance matrix in a central limit approximation are rewarded for reasons that may not be very appealing. In its most simple form, Ravi and I justified an estimation exercise based on minimizing pricing errors that led to a fixed choice of weighting matrix independent of the parameter value being considered.

Since misspecification can destroy the simple separation between estimation and decision, the aim of the parameter choice comes to the forefront. Ravi and I can be criticized for our ad hoc choice of loss function, but it is also sometimes unappealing to study moment relations in which the weighting matrix can change with the underlying parameter vector. Model misspecification is an example.

9. Editors: GMM has proven particularly valuable for the estimation of rational expectation models, because it facilitates estimation based on Euler equations without the need to impose strong explicit distribution assumptions. In recent years, you have turned your attention to joint research with Tom Sargent to robust decision problems. In this framework, agents are assumed to take model misspecification into account while making decisions. This comes at a cost of very explicit assumptions about the probability laws governing the economic 
environment and takes us away from the "distributionfree" approach of GMM-based Euler equation estimation. What are your thoughts on this development?

L.P.H.: GMM approaches based on Euler equations were designed to deliver only part of an economic model. Their virtue and liability is that they are based on partial specification of an econometric model. They allow an applied researcher to study a piece of a full dynamic model, without getting hung up on the details of the remainder of the model. For many purposes, including policy analysis and the conduct of comparative dynamic exercises, the entire model has to be filled out. We could require this full specification of all econometric exercises in applied time series, but I think this would be counterproductive. On the other hand, to solve decision problems by policy makers or to conduct comparative dynamic exercises a full decision problem, and a complete dynamic evolution must be specified. A GMM estimation of a portion of the model can be an input into this, but more is required.

Rational expectations compels a researcher to think about model specification both from the standpoint of the applied researcher and from the standpoint of individual decision makers. We could presume that when we fill out the entire model including the dynamic evolution of state variables, this is done correctly. Rational expectations becomes an equilibrium construct that compels the researcher to check for correct specification from all angles, econometrician and economic agents. It is a powerful and tremendously productive equilibrium concept.

The question is what do you do if you suspect misspecification. Interestingly, if you look at applied research in economic dynamics that imposes rational expectations, one of the reasons people give for not getting distracted by econometrics is that the model is obviously misspecified. Once you go down this road, however, you naturally ask from whose perspective. The consistency requirement of the equilibrium concept forces this misspecification on the economic agents, and the description "rational expectations" becomes a bit of a misnomer. There is no obviously simple fix to this problem. We could remove misspecification from the table as rational expectations does in such a clever way, but this seems incompatible with much applied research and arguably individual decision making.

The work you describe (with a variety of coauthors) is one stab at confronting model misspecification. Since we are compelled to put a rich class of models in play, typically in the form of an approximating or benchmark model and a family of perturbations, there are hard questions about which models should be put into contention and how they should be confronted by decision makers. We have been working on some tractable ways to approach model misspecification, building from a rich literature in robust control theory, but this is far from a mature literature. Our work to date has been very controversial, and some of this controversy is well justified. We are still sorting out strengths and weakness of alternative approaches in our own minds.

Our robustness approach is related more closely to Sargent's and my work (Hansen and Sargent 1993) and related work by Sims (1993) on estimation of fully specified dynamic rational expectations models using a misspecified likelihood function than it is to GMM estimation of partially specified models. Since we are compelled to work with well-posed decision problems, we need the entire model.

10. Editors: Over the last 20 years, there has been much progress in developing a framework for inference based on the GMM estimator. What do you perceive to be the strengths and weaknesses of the framework as it stands today?

L.P.H.: GMM estimation is often best suited for models that are partially specified. This is both a strength and a weakness. Partial specification is convenient for a variety of reasons. It allows an econometrician to learn about something without needing to learn about everything. An appeal to partial specification, however, limits the questions that can be answered by an empirical investigation. For instance, the analysis of hypothetical interventions or policy changes typically requires a fully specified dynamic, stochastic general equilibrium model. Applied researchers in macroeconomics and finance use highly stylized, and hence misspecified, versions of such models. Such models are often studied through numerical characterization for alternative parameter configurations. It remains an important challenge to econometrics to give empirical credibility to the estimation and testing of such models.

\section{REFERENCES}

Amemiya, T. (1974), "The Nonlinear Two-Stage Least Squares Estimator," Journal of Econometrics, 2, 105-110.

(1977), "The Maximum Likelihood and Nonlinear Three-Stage Least Squares Estimator in the General Nonlinear Simultaneous Equations Model," Econometrica, 45, 955-968.

Andrews, D. W. K. (1999), "Consistent Moment Selection Procedures for Generalized Method of Moments Estimation," Econometrica, 67, 543-564.

Billingsley, P. (1961), "The Lindeberg-Levy Theorem for Martingales," Proceedings of the American Mathematical Society, 12, 250-268.

Bonnal, H., and Renault, E. (2000), "A Comparison of Alternative Moment Matching Estimators," CREST-INSEE.

Brown, B. W., and Maital, S. (1981), "What Do Economists Know? An Empirical Study of Experts' Expectations," Econometrica, 49, 491-504.

Chamberlain, G. (1987), "Asymptotic Efficiency in Estimation With Conditional Moment Restrictions," Journal of Econometrics, 34, 305-334.

Clark, P. K. (1973), "A Subordinated Stochastic Process Model With Finite Variance for Speculative Prices," Econometrica, 41, 135-155.

Conley, T. G., Hansen, L. P., Luttmer, E. G. J., and Scheinkman, J. A. (1997), "Short-Term Interest Rates as Subordinated Diffusions," Review of Financial Studies, 10, 525-577.

Eichenbaum, M., and Hansen, L. P. (1990), "Estimating Models With Intertemporal Substitution Using Aggregate Time Series Data," Journal of Business and Economic Statistics, 8, 53-69. 
Godambe, V. P., and Heyde, C. C. (1987), "Quasi-Likelihood and Optimal Estimation," International Statistical Review, 55, 231-244.

Gordin, M. I. (1969), "The Central Limit Theorem for Stationary Processes," Soviet Math. Dokl., 10, 1174-1176.

Grossman, S. J., and Shiller, R. J. (1981), "The Determinants of the Variability of Stock Prices," American Economic Review Papers and Proceedings, 71, 222-227.

Hall, A. R., and Inoue, A. (2001), "A Canonical Correlations Interpretation of Generalized Method of Moments Estimation With Applications to Moment Selection," North Carolina State University, unpublished manuscript.

Hall, P., and Heyde, C. C. (1980), Martingale Limit Theory and Its Application, Boston: Academic Press.

Hall, R. E. (1978), "Stochastic Implications of the Life Cycle Permanent Income Hypothesis: Theory and Evidence," Journal of Political Economy, 86, 339-357.

Hansen, L. P. (1982), "Large Sample Properties of Generalized Method of Moments Estimators," Econometrica, 50, 1029-1054.

(1985), "A Method for Calculating Bounds on Asymptotic Covariance Matrices of Generalized Method of Moments Estimators," Journal of Econometrics, 30, 203-238.

(2002), "Method of Moments," in International Encylopedia of the Social and Behavior Sciences, Oxford, U.K.: Pergamon.

Hansen, L. P., Heaton, J., and Luttmer, E. G. J. (1995), "Econometric Evaluation of Asset Pricing Models," Review of Financial Studies, 8, 237-274.

Hansen, L. P., Heaton, J. C., and Yaron, A. (1996), "Finite Sample Properties of Some Alternative GMM Estimators," Journal of Business and Economic Statistics, 14, 262-280.

Hansen, L. P., and Hodrick, R. J. (1980), "Forward Exchange Rates as Optimal Predictors of Future Spot Rates," Journal of Political Economy, 88, 829-853.

Hansen, L. P., and Jagannathan, R. (1997), "Assessing Specification Errors in Stochastic Discount Factor Models," Journal of Finance, 52, 557-590.

Hansen, L. P., and Sargent, T. J. (1993), "Seasonality and Approximation Errors in Rational Expectations Models," Journal of Econometrics, 55, $21-55$.

Hansen, L. P., and Scheinkman, J. A. (1995), "Back to the Future: Generating Moment Implications for Continuous-Time Markov Processes," Econometrica, 63, 767-804.
Hansen, L. P., and Singleton, K. J. (1982), "Generalized Instrumental Variables of Nonlinear Rational Expectations Models," Econometrica, 50, $1269-1286$.

(1983), "Stochastic Consumption, Risk Aversion, and the Temporal Behavior of Asset Returns," Journal of Political Economy, 91, 249-265. (1991), "Computing Semiparametric Efficiency Bounds for Linear Time Series Models," in Proceedings of the Fifth International Symposium in Economic Theory and Econometrics, eds. W. A. Barnett, J. Powell, and G. E. Tauchen, Cambridge, U.K.: Cambridge University Press, chap. 15, pp. 388-411.

(1996), "Efficient Estimation of Linear Asset Pricing Models With Moving-Average Errors," Journal of Business and Economic Statistics, 14, 53-68.

MaCurdy, T. E. (1978), "Two Essays on the Life Cycle," unpublished doctoral dissertation, University of Chicago, Department of Economics.

Newey, W. K. (1990), "Efficient Instrumental Variables Estimation of Nonlinear Models," Econometrica, 58, 809-837.

Newey, W. K., and Smith, R. J. (2000), "Asymptotic Bias and Equivalence of GMM and GEL," unpublished manuscript.

Sargan, J. D. (1958), "The Estimation of Economic Relationships Using Instrumental Variables," Econometrica, 26, 393-415.

(1959), "The Estimation of Relationships With Autocorrelated Residuals by the Use of Instrumental Variables," Journal of the Royal Statistical Society, 21, 91-105.

Scott, D. J. (1973), "Central Limit Theorems for Martingales and for Processes With Stationary Increments Using a Skorokhod Representation Approach," Advances in Applied Probability, 5, 119-137.

Shiller, R. (1972), "Rational Expectations and the Structure of Interest Rates," unpublished doctoral dissertation, Massachusetts Institute of Technology.

Sims, C. A. (1993), "Rational Expectations With Seasonally Adjusted Data," Journal of Econometrics, 55, 9-19.

Soderstrum, T., and Stoica, P. G. (1983), Instrumental Variable Methods for System Identification, Berlin: Springer-Verlag.

Stock, J. H., and Wright, J. H. (2000), "GMM With Weak Identification," Econometrica, 68, 1055-1096.

West, K. D., and Wilcox, D. W. (1996), "A Comparison of Alternative Instumental Variables Estimators of a Dynamic Linear Model," Journal of Business and Economic Statistics, 14, 281-293. 